\title{
Visual Analytics for Semantic Queries of TerraSAR-X Image Content
}

\author{
Daniela Espinoza-Molina ${ }^{a}$, Kevin Alonso ${ }^{a}$ and Mihai Datcu ${ }^{a}$ \\ ${ }^{a}$ German Aerospace Center (DLR), Remote Sensing Technology Institute, Box 1116, 82230, \\ Oberpfaffenhofen, Germany;
}

\begin{abstract}
With the continuous image product acquisition of satellite missions, the size of the image archives is considerably increasing every day as well as the variety and complexity of their content, surpassing the end-user capacity to analyse and exploit them. Advances in the image retrieval field have contributed to the development of tools for interactive exploration and extraction of the images from huge archives using different parameters like metadata, key-words, and basic image descriptors. Even though we count on more powerful tools for automated image retrieval and data analysis, we still face the problem of understanding and analyzing the results. Thus, a systematic computational analysis of these results is required in order to provide to the end-user a summary of the archive content in comprehensible terms. In this context, visual analytics combines automated analysis with interactive visualizations analysis techniques for an effective understanding, reasoning and decision making on the basis of very large and complex datasets. Moreover, currently several researches are focused on associating the content of the images with semantic definitions for describing the data in a format to be easily understood by the end-user. In this paper, we present our approach for computing visual analytics and semantically querying the TerraSAR-X archive. Our approach is mainly composed of four steps: 1) the generation of a data model that explains the information contained in a TerraSAR-X product. The model is formed by primitive descriptors and metadata entries, 2) the storage of this model in a database system, 3) the semantic definition of the image content based on machine learning algorithms and relevance feedback, and 4) querying the image archive using semantic descriptors as query parameters and computing the statistical analysis of the query results. The experimental results shows that with the help of visual analytics and semantic definitions we are able to explain the image content using semantic terms and the relations between them answering questions such as what is the percentage of urban area in a region? or what is the distribution of water bodies in a city?
\end{abstract}

Keywords: data mining, knowledge discovery, semantic annotations, visual analytics

\section{INTRODUCTION}

The enormous amount of Earth Observation (EO) data contained in space agencies archives surpasses the enduser capacity to analyse and exploit them and requires new technologies and procedures to efficiently retrieve and visualize the information of interest. In the specific field of Earth Observation, along the years several solutions for searching and retrieving EO images have been presented. Initially there were tools that offer product catalogues, which allow to make queries based on satellite/instrument characteristics, acquisition dates, geographic coordinates. ${ }^{1}$ Later with the introduction of the concept "query by example" several implementations of search engines using the image content as query parameter were presented as for example the Knowledge based Information retrieval (KIM) system, ${ }^{2}$ and the Geospatial Information Retrieval and Indexing System (GeoIRIS). ${ }^{3}$ In the next generation of search engines, semantic concepts were integrated to the image content in order to make stronger the retrieval and to partially solve the semantic gap caused by the different understanding between humans and machines. ${ }^{4,5}$ Presently, geo-information plays an important role in finding scenes of interest and projecting the results on a global view as a map representation. Thus, the tendency is to use geospatial information for querying and visualizing the content of image archives. For instance, the implementation of threetier system for effectively visualize and query geospatial data was presented in Ref. ${ }^{6}$ This system simulated geolocations and fusion relevant geospatial data in a virtualized model in order to support decisions. In the

Further author information: E-mail: daniela.espinozamolina@dlr.de 
work of $\mathrm{Li}^{7}$ the geotags and the underlying geocontext for an advanced visual search were exploited showing satisfactory results in the image retrieval. It has been mentioned ${ }^{8}$ the importance of integrating a geospatial infrastructure based on standardized web services into an EO data library, where the design of harmonized data and information models of the EO and geospatial community is a precondition for interoperability at metadata, data and semantic levels.

Even though we currently count on more powerful tools for automated image retrieval and data analysis based on data mining and knowledge discovery methods, we still face the problem of understanding and analysing the results. As for example the lack of tools for data analytics was discussed in $^{9}$ where the example of NLCD database was exposed, here the database has not been analyzed so far due to the lack of tools beyond basic statistics and SQL queries; thus, the authors introduced a new application called Land-ExA, which is a Geoweb tool for query and retrieval of spatial patterns inland cover datasets. Therefore, now the challenge is how to visualize the results in a way that can be easily understood by different types of final users. Methods like data visualization, visual data exploration, and visual analytics play an important role in the data mining process and presentation of query results. The importance of integration of interactive geospatial data visualization with statistical data mining algorithms was highlighted in Ref. ${ }^{10}$ showing an application of visual data mining to visualize geospatial data. The same authors mention that the goal of visual analytics research is to turn the information overload into an opportunity by enabling decision-makers to examine this massive information stream to take effective actions in real-time situation. ${ }^{11}$

In this paper, we are concerned with computing different analytics of the image archive together with auxiliary information coming from geonames database and in-situ data. In order to compute analytics about the image content is necessary the association of the image content with semantic definitions, this is achieved by using machine learning methods. The present paper is organized as follows. In Section 2, a description of work flow and the architecture of the tools used in this paper are presented. Section 3 describes the semantic definition via machine learning methods and relevance feedback. Section 4 outlines the test data sets and describes the results of the computation of some analytics. Finally, Section 5 gives some conclusions.

\section{DESCRIPTION OF THE ARCHITECTURE}

Figure 1 provides the overall system structure and its components. The details of each block are presented in Refs. ${ }^{12,13}$ The system consists of several modules, which will interact to provide the required data flow and functionality for the data ingestion, the semantic content generation that enriches the standard metadata and makes the enhanced set of metadata available to the end user for querying and computing analytics of the image archive. We distinguish three kinds of system users, the ground segment system administrator (operator), the EO expert, and the end-user. Each of them will interact with the system in a different manner and in consequence they will access different modules of the overall system. The ground segment system administrator will oversee and perform data ingestion and maintenance of the data sources. He will therefore access internal system components. These components are depicted to the upper-left part of Figure 1. The expert will define the semantic links. By means of interactive learning, he will link the image content with standard metadata and semantic annotations. End users will access the system via the user interface depicted on the lower-left side of Figure 1. They will use the enhanced metadata, provided by the system, to perform advanced content-based image retrieval and semantic definition. These advanced querying and data exploration capabilities, provided by knowledge discovery component and by the query engine, will be used as search and discovery tools for computing analytics of the image content.

Figure 1 also shows a high-level workflow of the system prototype. Here, the upper part summarizes the Data Model Generation (DMG), the middle part describes the Knowledge Discover in Databases (KDD), and the lower part illustrates the Query Engine (QE). The data model generation tool is in charge of the content analysis of the EO sources. That includes tiling the images to generate multi-size and multi-resolution patches; extracting the metadata from the sources, converting the patch content into primitive features, and storing all the processed data into the database. The DMG input is an EO image, which is either multi-spectral or Synthetic Aperture Radar (SAR), and its processing parameters. The DMG output is the image content descriptors in the form of vectors (e.g., feature vectors) together with high resolution quick-looks, and metadata entries. The DMG functionalities are: 1) prepare and set the ingestion of EO products, 2) extract the metadata, 3) generate the 


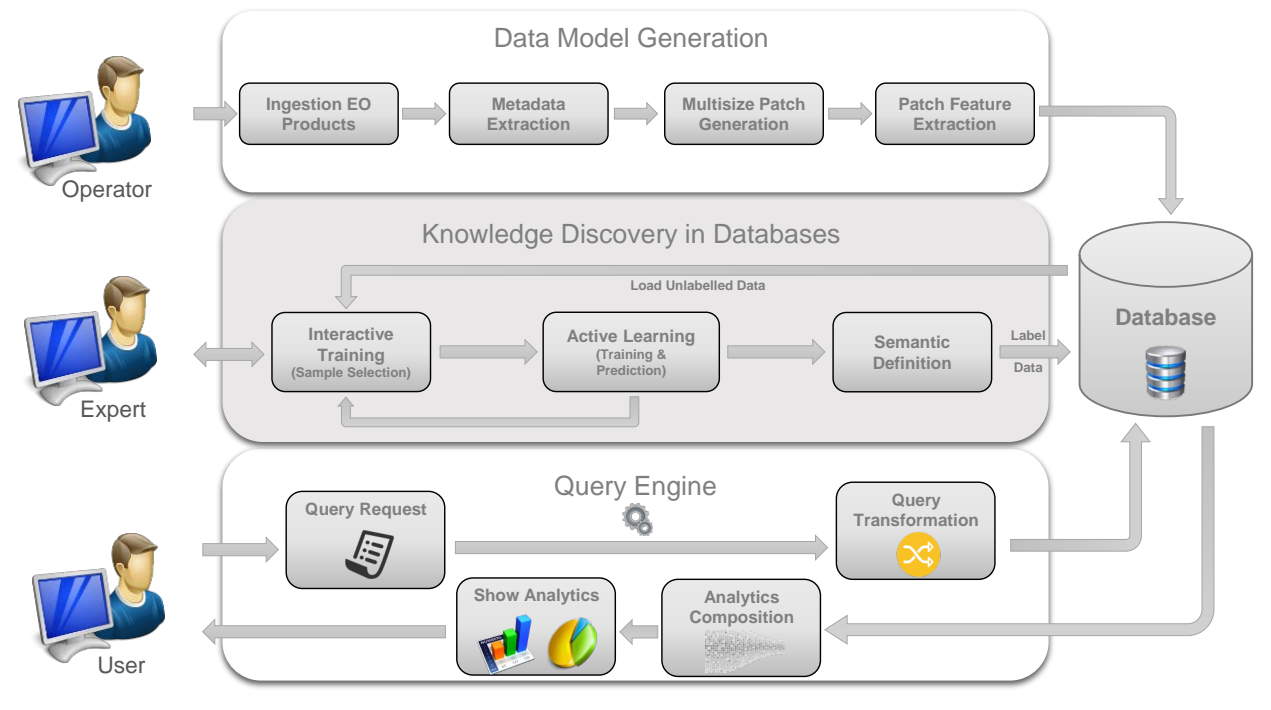

Figure 1. Overall system structure and the interaction between its components

quick-look of the patches, 4) extract the primitive feature using two methods, Gabor filters ${ }^{14}$ and Weber local descriptors, ${ }^{15}$ and 5) store all the processed information into the database scheme. The workflow of the data model generation is shown in the upper part of Figure 1. DMG starts with creating a collection and specifying the EO products to be ingested together with their input parameters (i.e. product path, patch size, levels of resolution, etc.). Here, the term collection describes a set the EO products with the same purpose and type i.e., either optical images or SAR images. In the next step the metadata content analysis component reads the annotation file, which comes in a XML format, and extracts the relevant metadata entries (e.g. incidence angles, coordinates, sensor type, etc.). Further, the image content analysis component cuts the EO products generating a grid of multi-size patches with their quick-looks. Following, the primitive features are extracted by the selected methods. Finally, all the generated information is stored into the database. It is important to mention that during the entire workflow of the data model generation, the data managed by the system is transformed, passing from an initial form of full EO products to much smaller added value products (image descriptors, metadata entries, etc), called data model, which will be later available for immediate exploitation and analysis by the users.

The middle part of Figure 1 describes the semantic definition based on active learning methods. The Knowledge Discovery in Databases (KDD) module has available all the features, patches and related information stored in the database, this module accesses to the repository to get relevant information for supporting its functionalities like query by example, data mining, and semantic definition of the image content. These functionalities offer an integrated tool that exploits intensively the user interaction to perform advanced content-based image retrieval and semantic label definition. The definition of semantic concepts for images is an exhausting and expensive task. In our approach, the image content is semantically annotated using the Support Vector Machine (SVM) as active learning methods and the user interaction. ${ }^{16}$

While the passive model of supervised learning all the labels are obtained without reference to the learning algorithm, in active learning the learner interactively chooses which data points to label. The ambition of active learning is that interaction can substantially reduce the number of labels required, making solving problems via machine learning more practical. ${ }^{17}$ In the KKD module, the active learning algorithm uses a large pool of unlabelled data (test data) and only a small set of labelled data (training data). The expert (end-user) defines the training data to be used by giving positive and negative examples. A positive example means that the patch contains the desired content. Active learning has two core components the selection strategy and model learning, which are repeated until convergence. At the beginning of the procedure, only a few labelled instances are available (training data) then a coarse classifier is learnt and applied to the test data. After that, the iteration of the two components is repeated until the classification results are satisfactory. In each iteration, most informative samples from the unlabelled instances in the data set are selected and labelled manually (selected 
by the expert as positive or negative examples) to improve the current classifier. These new labelled instances are added to the training set and a new classifier is learnt again. This process is repeated several times until the expert is satisfied with the classification results, then a new semantic label is assigned to the classified data set and the new labelled data is stored in the database.

The lower part of Figure 1 depicts the Query Engine, which is used to make several queries using various parameters like metadata, feature vectors, semantic labels. This tool accepts the user entries and transforms the requests to a standard query language in order to retrieve the information from the database. Following, the results are shown to the user as pie charts, linear plots, etc. making possible a visual analytics.

\section{SEMANTIC DEFINITION OF THE IMAGE CONTENT}

There are several approaches for assigning semantic descriptions to the image content. In a study conducted by ${ }^{18}$ the annotation or large satellite images is based on supervised classification of the patches and the integration of spatial information between the patches. In this work, the semantic concepts are defined by the user. In Ref. ${ }^{19}$ proposed the image annotation by using a game which includes semantic structure by means of the WordNet ontology. Our approach follows the recommendations of Ref., ${ }^{20}$ where the authors presented the taxonomies that can be retrieved from TerraSAR-X products. Here the semantic categories are grouped in a two-level hierarchical taxonomy, where the main categories describe general land use and land cover classes (i.e. urban area, water bodies, forest, bare ground, agriculture), while the secondary categories represent specific characteristics of the main categories as for example high density urban area, industrial areas, forest, tress, lakes, see, ocean, etc. The complete taxonomy is fully described in. ${ }^{20}$ Figure 2 shows examples of the diversity of TerraSAR-X image content, the hierarchical organization of the semantic taxonomy with two level categories, and a patch example per category. Starting from left to right part of the Figure 2, the images are annotated as high density residential area, industrial area, bridge, road, stubble, crops, coniferous, trees, sand, beach, lake, and river. It is important to observe how the different structure are represented in TerraSAR-X and only using a patch approach is possible to have the context of the scene for a proper annotation of the image content.

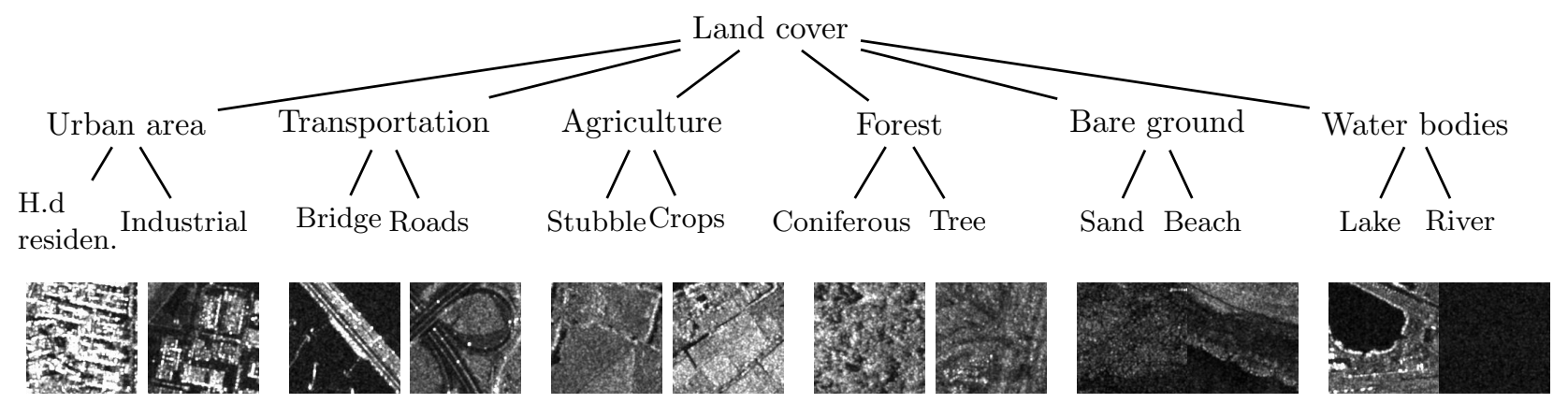

Figure 2. Example of the semantic catalogue of TerraSAR-X image content. (Left-right) High density residential area, industrial area, bridge, road, stubble, crops, coniferous, trees, sand, beach, lake, and river.

The adopted methodology ${ }^{21}$ to annotate the test data starts grouping the image products into collections using the DMG tool previously presented. Later, these products are cut into patches with $160 \times 160$ pixels size, the high resolution quick-looks of each patch are generated and the gabor and weber primitive features associated to these patches computed. To finalize the ingestion all the generated information is stored into the database. Once the ingestion is completed, we use the KDD tool to annotate the image content. The KDD tool supports the users to search patches of interest in a large repository. Through the Graphical User Interface (GUI) of this tool, a list of patches with their respective quick look are shown to the user. The KDD allows to rank the suggested images which are expected to be grouped in a class of relevance. When a relevant class is found with the help of human expertise, the annotation of this set of patches is generated giving a semantic meaning to the retrieved classes and grouping the patches accordingly. In order to have a better accuracy of the classification results, the content of the patches is verified in Google Earth. 


\section{EXPERIMENTAL RESULTS}

\subsection{Overview of the data set}

The test data set is composed of Synthetic Aperture Radar (SAR) images, specifically TerraSAR-X (TSX) data, ${ }^{22}$ in-situ data taken from LUCAS (Land Use/Cover Area frame statistical Survey), ${ }^{23}$ and a database formed of millions of points with geographic location and semantic definitions called geonames.

\subsubsection{TerraSAR-X data set}

The SAR image database consists of 364 TerraSAR-X L1b products comprising around 28490 metadata entries. From the variety of TSX product types, we selected Multi-look Ground range Detected (MGD) products because they are not affected by the interpolation and are more suitable for image analysis. The characteristics of the TerraSAR-X dataset are: Radio metrically enhanced (RE) Multi-look Ground range Detected (MGD) products, in high resolution spotlight mode with pixel spacing equals to 1.25 meters, and resolution of about 2.9 meters, includes ascending and descending pass directions with single polarization $\mathrm{HH}$ or VV. These images were tiled resulting in 406277 very high resolution patches of $160 \times 160$ pixels. Each patch is characterized by a primitive feature (PF) vector and a semantic annotation. A total of 100 TSX scenes were annotated resulting in 157661 annotations with around 300 semantic labels. Figure 3 illustrates the distribution of the test data set around the world. The green points represent the location of the different scenes. The largest number of scenes was taken over Europa. However scenes over Asia, America and Africa were also included.

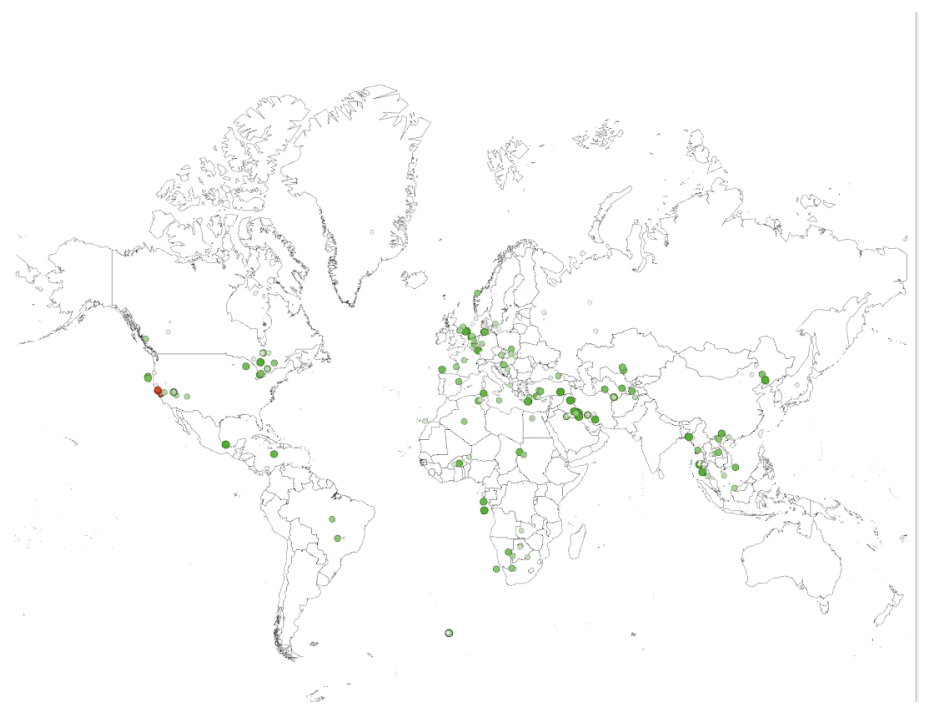

Figure 3. Distribution of the test TerraSAR-X scenes around the world. The green points represent the localization of the scenes.

\subsubsection{LUCAS data set}

LUCAS is an effort of the European Commission who has carried out a survey on the state and the dynamics of changes in land use and cover in the European Union during 2006 to 2013. LUCAS surveys are carried out in situ, which means that the data is gathered through direct observations by the surveyors on the ground all over the European Union. A surveyor recorded the geographical information (latitude/longitude coordinates) and the thematic information such as the content of the terrain (grass, crops, etc.). In addition, a photograph sequence was taken for each location successively looking at North, East, South, and West (clockwise rotation). LUCAS land cover classification is a 3 level hierarchical scheme. The main level contains 8 land cover categories: artificial land, cropland, woodland, shrub land, grassland, bare land, water and wetland. In total, there are 84 categories for land cover. Our experiments were conducted using LUCAS survey over Germany in 2009, which is composed of about 22.000 observed points, 95.000 photos with $1600 \times 1200$ pixel size, and 84 land use/land 
cover categories. Figure 4 shows examples of Lucas photos at different locations, the observed point is marked with a red flag. The photos were taken in the four cardinal directions.

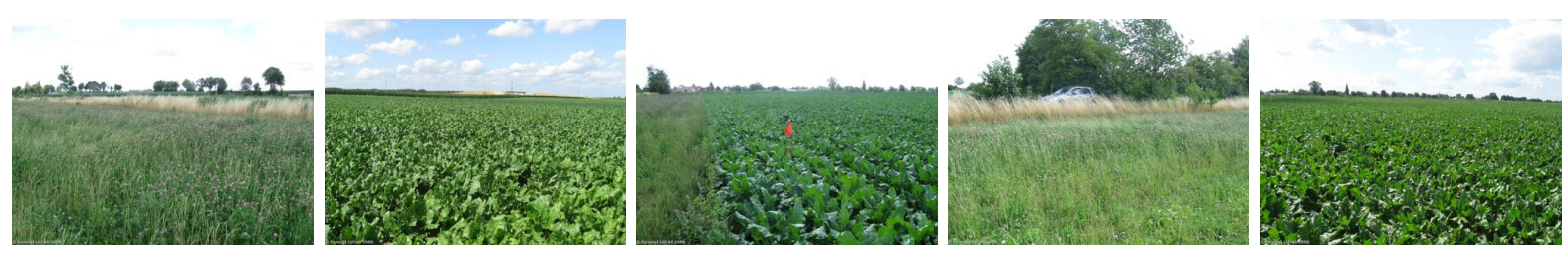

Figure 4. Example of LUCAS in-situ photos considering (left-right) North, West, Observed Point, East and South directions at the observed point.

\subsubsection{GeoNames}

GeoNames is a gazetteer that collects both spatial and thematic information for various place names around the world. It contains over 10 million geographical names and consists of over 8 million unique features whereof 2.8 million populated places and 5.5 million alternate names. All features are categorized into one out of nine feature classes and further sub-categorized into one out of 645 feature codes. GeoNames integrates geographical data such as location names in various languages, elevation, population and others from various sources. In our test we restrict the geonames data set to Europa and the use of continent names.

\subsection{Examples of Analytics}

The following sub sections present some examples of analytics based on land cover and land use distribution. The analytics helps the end-user to better understand the content of data and the relation between different variables. Examples are land cover, expressed in semantic categories, with geographical localization, or image resolution and semantic categories according to the incidence angles.

\subsubsection{Analytics using metadata and semantic categories based on SAR data}

In order to understand the relationship between the image resolution with the incidence angle and the total number of annotation per continents, we compute a query in our database involving metadata entries (angle, resolution), with localization (geographical coordinates) and semantic categories ( total of annotations). Figure 5 shows the relationship between incidence angle and resolution in the six continents. The total number of annotations is represented by the size of the bubbles. For example, we can see that the largest number of annotation corresponds to south America, and they are between 22 and 24 degrees with more than $2.5 \mathrm{~m}$ of resolution. The scenes corresponding to Europa are spread around 34 and 50 degrees, whereas the scenes in Africa can be found at 40 to 42 degrees. Europa has more scenes annotated. The number of annotations between Asia and Africa is similar.

A second example is plot in Figure 6, it shows the distribution of the main land use land cover categories around the world. In this case metadata entries, semantic annotations and geographical locations are used. Here, it can be observed that the largest land cover in our test data set corresponds to Bare Ground category in South America. The highest amount of Forest is located in Europa, whereas the lowest amount of forest is in Africa. In North America there is the largest amount of Urban Area. Asia has the biggest number of annotations corresponding to water bodies semantic category. Finally, agriculture has the lowest number of annotations.

Figure 7 shows a comparison of some land cover land use categories in different cities around the world. Here it can be observed that cities like Madrid in Spain has the highest number of Roads, while Bogota in Colombia has the lowest number of Roads. We can remark that Sand semantic category appears only in Saudi Arabia and Iran. Regarding the number of Skyscrapers is almost homogeneously distributed between Ottawa in Canada, Kuala Lumpur - Malasia, and San Francisco in USA. Moreover, the category Forest mixed highly annotated in Singapore.

Analytics of land cover distribution of Germany according to TerraSAR-X annotations is presented in Figure 8. This graphic was done using Gephi tool. ${ }^{24}$ Figure 8 shows the distribution of the land cover in eleven German 


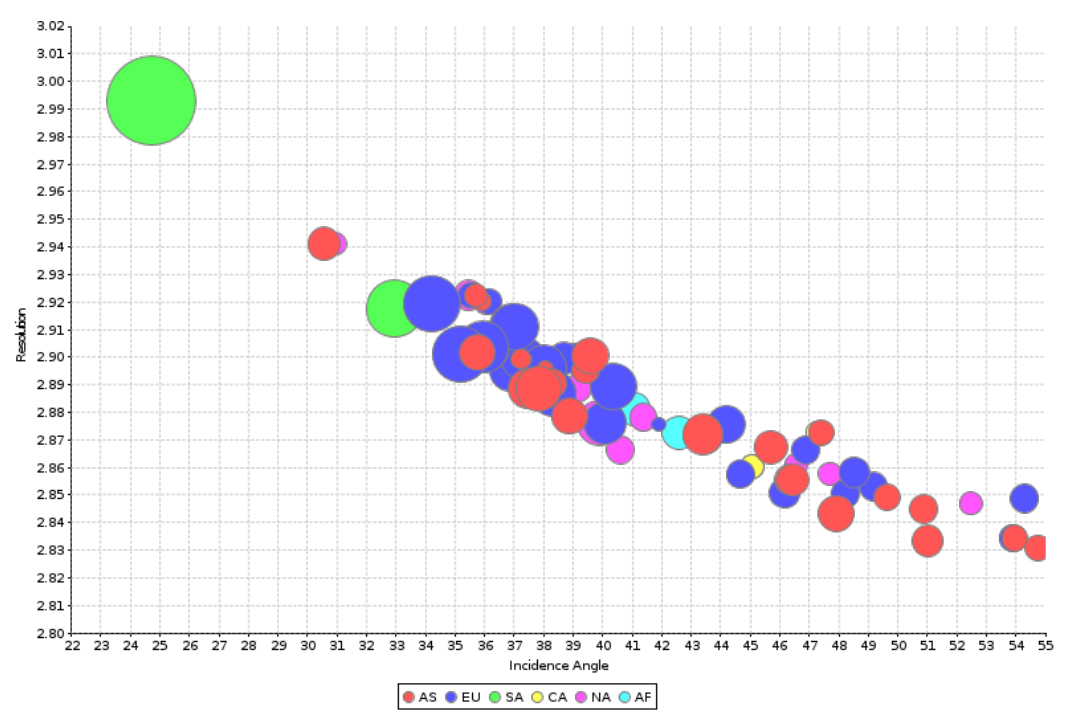

Figure 5. Incidence angle versus ground range resolution and total number of annotations per continent used as test database. $\mathrm{AS}=\mathrm{Asia}, \mathrm{EU}=$ Europa, $\mathrm{SA}=$ South America, $\mathrm{CA}=$ Central America, NA=North America, AF=Africa.

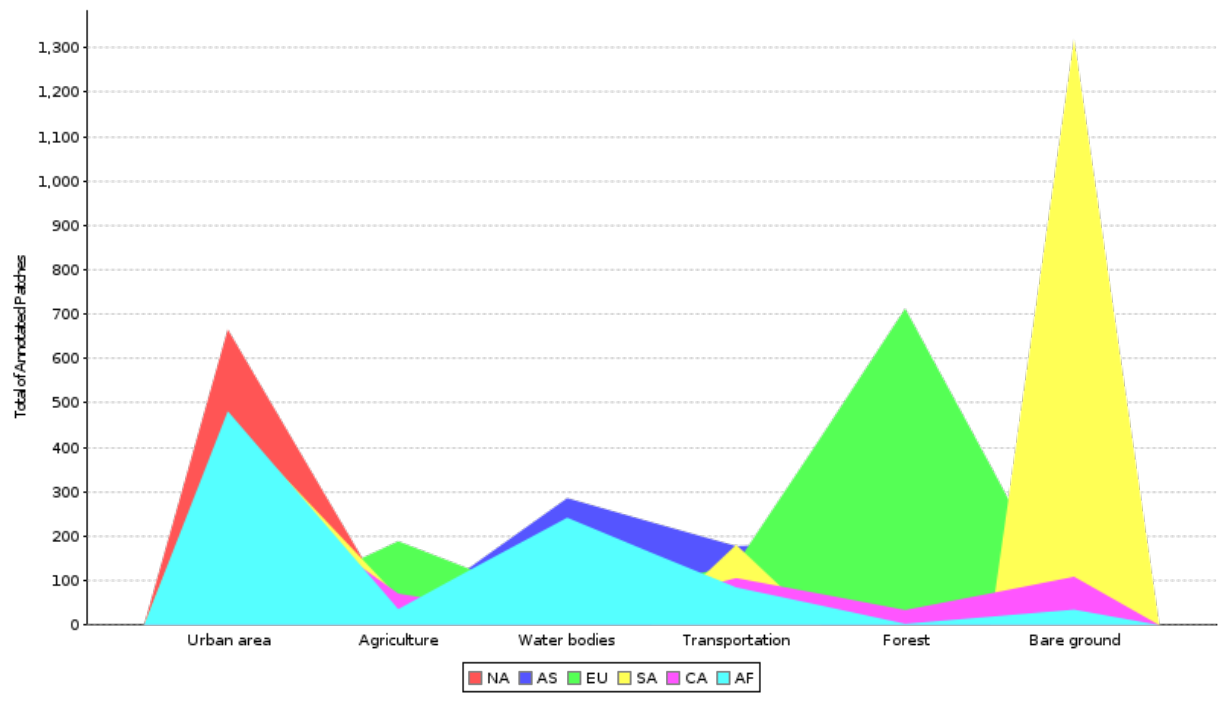

Figure 6. Distribution of main Land Cover Land Use categories around the world. AS= Asia, EU=Europa, SA=South America, $\mathrm{CA}=$ Central America, NA=North America, and AF=Africa.

cities (Berlin, Bonn, Bremen, Cologne, Karlsruhe, Kiel, Lindau, Mannheim, Munich, Oldenburg and Stuttgart). Each axis represents a city. The land cover categories are marked in different colors as shows the legend. The size of the circles depends on the total number of annotations. In the figure can be observed that Berlin has the highest number of annotations corresponding to urban area, while Lindau has highest number of annotations with water bodies.

\subsubsection{Analytics using Land Use Land Cover categories based on in-situ data}

The distribution of Lucas dataset over Germany is shown in Figure 9. Here, the Lucas points are plot according to their geographical localization. The diversity of the semantic catalogue has around 84 land use and land cover categories. In this figure the total number of semantic annotations associated with the point were computed and summarized in the legend. As we can observed shown the most common land covers are Grassland (29.66 percent), Common wheat (13.05 percent) and Maize (9.75 percent). 


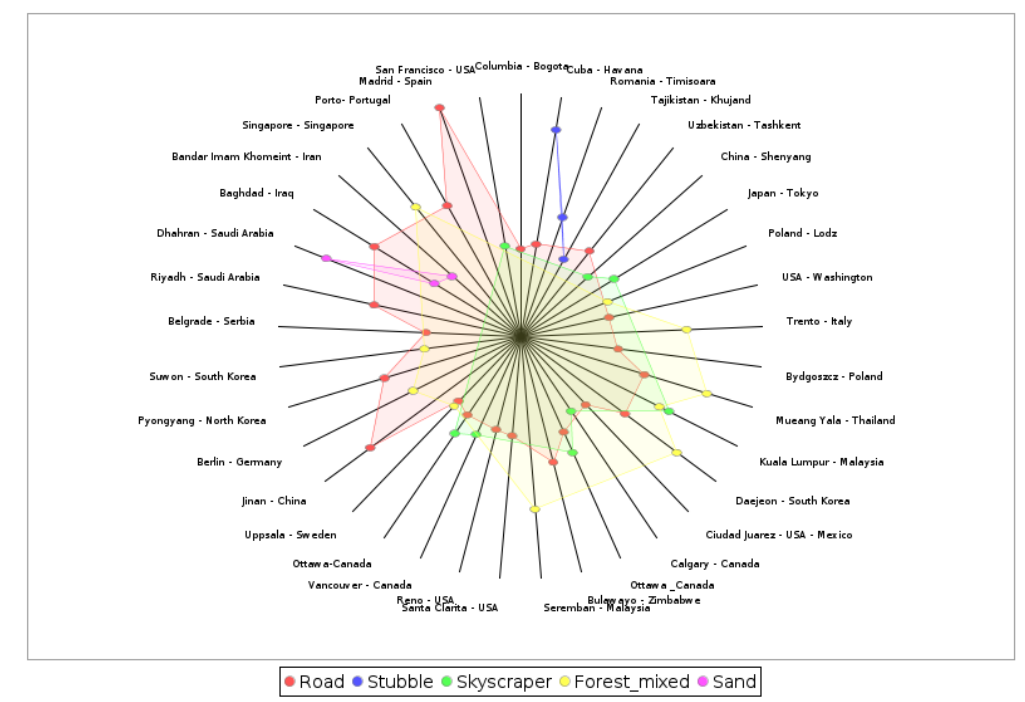

Figure 7. Distribution of Land Cover over Germany using Lucas database

Agricultural land

residential area-and-1

High building-and-1

Stubble-and-1

Road

Forest mixed

Bridge

Hiah buildina-and-2

residential area-and-1

and-Agricultural land

Storage tank

Road-and-1

Forest broadle

Railway-and-2

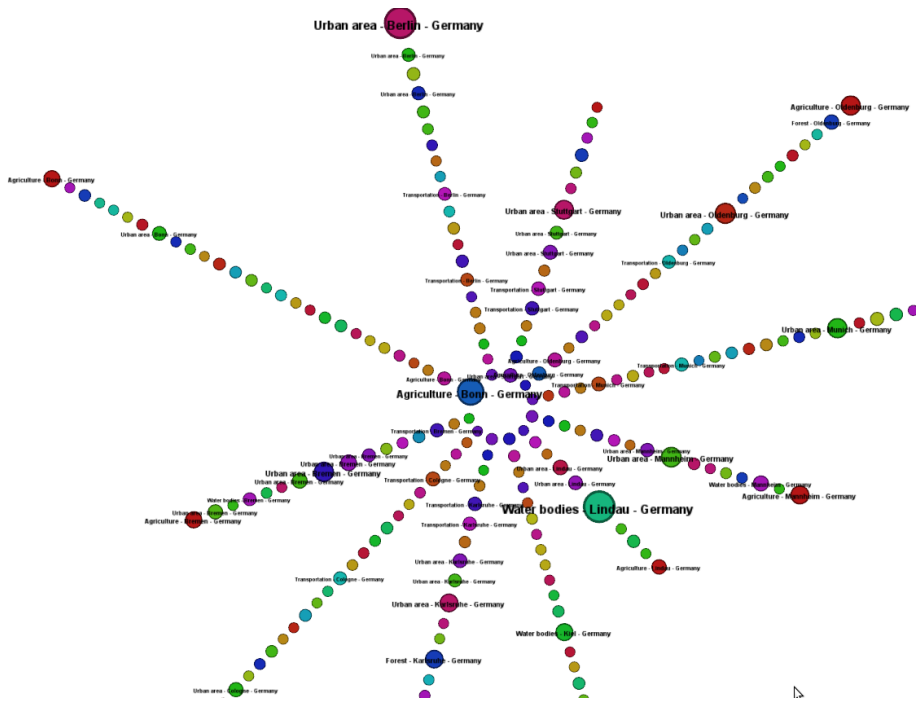

Figure 8. Distribution of Land Cover over Germany using TerraSAR-X data 

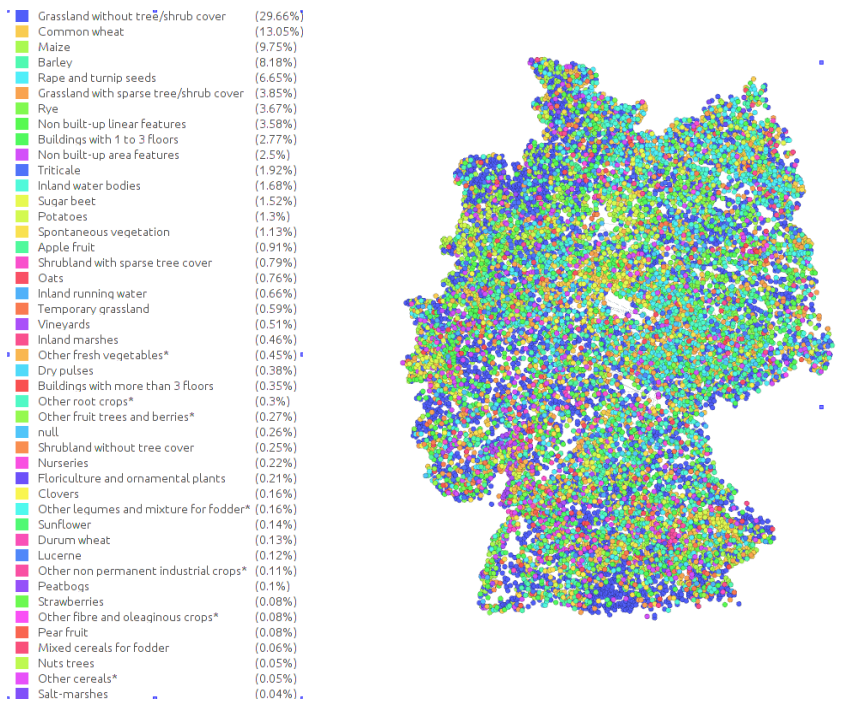

Figure 9. Distribution of Land Cover over Germany using LUCAS database

\section{CONCLUSIONS}

In this paper we presented some visual analytics for semantic queries of TerraSAR-X image content. Two main points were discussed, the semantic definition of the image content by means of active learning methods, and the computation of queries and analytics. We described the tools used for processing the EO images together with auxiliary data in order to have the features vectors that will be used in the knowledge discovery tool for annotation the image content. The experiments were carried out on about 364 TerraSAR-X scenes, where 100 scenes were annotated forming a semantic catalogue composed of 800 entries. The presented experiments concluded that visual analytics helps the end user to summarized the query results in a way that can be used for supporting decisions. Visualizations like the ones presented allows the end users to better understand the content of the image archive at the same time that summarizes it.

\section{REFERENCES}

[1] Wolfmüller, M., Dietrich, D., Sireteanu, E., Kiemle, S., Mikusch, E., and Böttcher, M., "Data Flow and Workflow Organization- The Data Management for the TerraSAR-X Payload Ground Segment," IEEE Transactions on Geoscience and Remote Sensing 47, 44 -50 (Jan. 2009).

[2] Datcu, M., Daschiel, H., Pelizzari, A., Quartulli, M., Galoppo, A., Colapicchioni, A., Pastori, M., Seidel, K., Marchetti, P., and D'Elia, S., "Information mining in remote sensing image archives: system concepts," IEEE Transactions on Geoscience and Remote Sensing 41, 2923 - 2936 (Dec. 2003).

[3] Shyu, C.-R., Klaric, M., Scott, G. J., Barb, A. S., Davis, C. H., and Palaniappan, K., "GeoIRIS: Geospatial Information Retrieval and Indexing System-Content Mining, Semantics Modeling, and Complex Queries," IEEE Transactions on Geoscience and Remote Sensing 45, 839-852 (Apr. 2007).

[4] Rasiwasia, N., Moreno, P., and Vasconcelos, N., "Bridging the Gap: Query by Semantic Example," IEEE Transactions on Multimedia 9, 923 -938 (Aug. 2007).

[5] Espinoza-Molina, D. and Datcu, M., "Earth-Observation Image Retrieval Based on Content, Semantics, and Metadata," IEEE Transactions on Geoscience and Remote Sensing 51, 5145-5159 (Nov. 2013).

[6] Shahabi, C., Banaei-Kashani, F., Khoshgozaran, A., Nocera, L., and Xing, S., "GeoDec: A Framework to Visualize and Query Geospatial Data for Decision-Making," IEEE MultiMedia 17, 14-23 (Jul. 2010).

[7] Li, X., Snoek, C. G. M., Worring, M., and Smeulders, A. W. M., "Fusing concept detection and geo context for visual search," in [Proc. 2nd ACM International Conference on Multimedia Retrieval], ICMR '12, 4:14:8, ACM, New York, NY, USA (2012). 
[8] Heinen, T., Kiemle, S., Buckl, B., Mikusch, E., and Loyola, D., "The Geospatial Service Infrastructure for DLR's National Remote Sensing Data Library," IEEE Journal of Selected Topics in Applied Earth Observations and Remote Sensing 2, 260-269 (Dec. 2009).

[9] Stepinski, T., Netzel, P., and Jasiewicz, J., "LandEx-A GeoWeb Tool for Query and Retrieval of Spatial Patterns in Land Cover Datasets," IEEE Journal of Selected Topics in Applied Earth Observations and Remote Sensing 7, 257-266 (Jan. 2014).

[10] Keim, D., Panse, C., Sips, M., and North, S., "Visual data mining in large geospatial point sets," IEEE Comput. Graph. Appl. 24(5), 36-44 (2004).

[11] Keim, D. A., Mansmann, F., Stoffel, A., and Ziegler, H., "Visual analytics," Encyclopedia of Database Systems , 1-6 (2009).

[12] Espinoza-Molina, D. and Datcu, M., "Data mining and knowledge discovery tools for exploiting big earth observation data," ISPRS: International Society for Photogrammetry and Remote Sensing to be published (2015).

[13] Espinoza-Molina, D., Dumitru, C. O., Datcu, M., and members, C., "Kdd prototype - phase i," tech. rep., German Aerospace Agency (Issue: 3.2.1).

[14] Manjunath, B. S. and Ma, W. Y., "Texture features for browsing and retrieval of image data," IEEE Transactions on Pattern Analysis and Machine Intelligence 18, 837-842 (Aug. 1996).

[15] Cui, S., Dumitru, C., and Datcu, M., "Ratio-Detector-Based Feature Extraction for Very High Resolution SAR Image Patch Indexing," IEEE Geoscience and Remote Sensing Letters 10, 1175-1179 (Sep. 2013).

[16] Cui, S., Dumitru, O., and Datcu, M., "Semantic annotation in earth observation based on active learning," International Journal of Image and Data Fusion, 1-23 (2013).

[17] Settles, B., "Active learning," Synthesis Lectures on Artificial Intelligence and Machine Learning 6(1), 1-114 (2012).

[18] Lienou, M., Maitre, H., and Datcu, M., "Semantic Annotation of Satellite Images using Latent Dirichlet Allocation," IEEE Geoscience and Remote Sensing Letters 7(1) (2010).

[19] Steggink, J. and Snoek, C. G. M., "Adding semantics to image-region annotations with the name-it-game," Multimedia Systems , 367-378 (2011).

[20] Dumitru, C. O., Shiyong Cui, G. S., and Datcu, M., "A taxonomy for high resolution sar images," in [Image Information Mining: Geospatial Intelligence from Earth Observation Conference (ESA-EUSC-JRC 2014)], 89-92 (2014).

[21] Dumitru, C. O., Datcu, M., Koubarakis, M., Sioutis, M., Nikolaou, B., and members, C., "Ontologies for the visual observatory for terrasar-x data," tech. rep., German Aerospace Agency (Issue: 6.2.1).

[22] DLR, [TerraSAR-X Ground Segment Basic Product Specification Document, TX-GS-DD-3302] (Oct. 2013). http://sss.terrasar-X.dlr.de/pdfs/TX-GS-DD-3302.pdf.

[23] commission, E., "Land Cover Use statistics: LUCAS," (2000). http://epp.eurostat.ec.europa.eu/portal/page/portal/lucas/

[24] Bastian, M., Heymann, S., and Jacomy, M., "Gephi: An open source software for exploring and manipulating networks," (2009). 